\title{
The Accuracy of Prostate Cancer Localization Diagnosed on Transrectal Ultrasound-Guided Biopsy Compared to 3-Dimensional Transperineal Approach
}

\author{
Kevin Krughoff, Khadijah Eid, Jason Phillips, Diliana Stoimenova, Daniel Smith, \\ Colin O'Donnell, E. David Crawford, and Al Barqawi \\ Department of Surgery, Division of Urology, University of Colorado Denver School of Medicine, Academic Office One Building, \\ Mail Stop C-319, 12631 East 17th Avenue, Room L15-5602, Aurora, CO 80045, USA
}

Correspondence should be addressed to Al Barqawi; al.barqawi@ucdenver.edu

Received 26 October 2013; Revised 3 December 2013; Accepted 11 December 2013

Academic Editor: Fabio Campodonico

Copyright (C) 2013 Kevin Krughoff et al. This is an open access article distributed under the Creative Commons Attribution License, which permits unrestricted use, distribution, and reproduction in any medium, provided the original work is properly cited.

\begin{abstract}
Background. Prostate cancer is often understaged following 12-core transrectal ultrasound- (TRUS-) guided biopsies. Our goal is to understand where cancers are typically missed by this method. Methods. Transperineal 3-dimensional mapping biopsy (3DMB) provides a more accurate depiction of disease status than transrectal ultrasound- (TRUS-) guided biopsy. We compared 3DMB findings in men with prior TRUS-guided biopsies to determine grade and location of missed cancer. Results were evaluated for 161 men with low-risk organ confined prostate cancer. Results. The number of cancer-positive biopsy zones per patient with TRUS was $1.38 \pm 1.21$ compared to $3.33 \pm 4.06$ with $3 \mathrm{DMB}$, with most newly discovered cancers originating from the middle lobe and apex. Approximately half of all newly discovered cancerous zones resulted from anterior 3DMB sampling. Gleason upgrade was recognized in 56 patients using 3DMB. When both biopsy methods found positive cores in a given zone, Gleason upgrades occurred most frequently in the middle left and right zones. TRUS cancer-positive zones not confirmed by 3DMB were most often the basal zones. Conclusion. Most cancer upgrades and cancers missed from TRUS biopsy originated in the middle left zone of the prostate, specifically in anterior regions. Anterior sampling may lead to more accurate diagnosis and appropriate followup.
\end{abstract}

\section{Introduction}

An increasing proportion of prostate cancer is being diagnosed at clinically insignificant stages, raising controversy surrounding overdiagnosis and overtreatment of a growing number of patients with potentially nonmortal yet psychologically morbid disease [1]. Data seem to suggest that Watchful Waiting (WW) or Active Surveillance protocols (AS) should be the method of choice in many or even most low-risk situations due to the long natural history of prostate cancer and the generally favorable prognosis for clinically localized noninvasive disease [2-4]. There now exists substantial evidence that an observational approach would be a practical, cost saving, and quality-of-life preserving option for many [5].

The proportion of men with clinically localized prostate cancer (LPC) who could potentially benefit from AS has risen from approximately $25 \%$ in the early 1990 s to almost $50 \%$ as of 2007 [6]; however the proportion of men on AS has remained largely unchanged for over a decade. Only $6.8 \%$ of men with prostate cancer opt for AS according to the CaPSURE database. This level has fluctuated somewhat over the years but has changed only slightly since 2000 when it was at $6.2 \%[6,7]$. The vast majority of low risk patients pursue surgical, radiological, and hormonal interventions, and this carries substantial implications on patient quality of life and the healthcare system as a whole given the significant risks of urinary and sexual side effects that come with these treatment modalities [8].

Poor AS enrollment may be attributable to limitations in our ability to evaluate the true extent of cancer at the individual level and predict progression [9]. Large and Eggener concluded after studying data pertaining to AS that more accuracy in identifying cancer grade and stage upon 
diagnosis would help AS gain more widespread acceptance [10]. Supporting Large and Eggener's conclusion is an analysis of radical prostatectomy specimens from men who were previously on AS, finding that most cancer progression was a result of undersampling during the initial biopsy [11]. In a more recent review of the advances in patient selection methods and treatment triggers for AS, Lees et al. also reported that more sophisticated biopsy schemes could have serious implications for advancing patient selection in AS [12].

One such biopsy scheme described by Lees et al. is a comprehensive 3-dimensional mapping biopsy (3DMB) procedure developed by Barqawi et al. that demonstrates substantial improvements in accuracy over the standard transrectal ultrasound guided (TRUS) biopsy. 3DMB is a comprehensive biopsy of the whole prostate gland. The prostate is placed within a virtual 3D grid and all biopsies are taken systematically at $5 \mathrm{~mm}$ interval in $x / y / z$-registered coordinates. Overall the risk of missing any cancer in any of the prostate regions is significantly lower than that reported for TRUS biopsy which is around $25-30 \%$ [13]. Their initial report on the use of $3 \mathrm{DMB}$ found that a significant portion of 180 men diagnosed with low-risk disease harbored clinically significant cancers, resulting in approximately a quarter of them receiving an upgraded cancer diagnosis and half receiving an upstaged diagnosis [13].

Since its inception, researchers have demonstrated the ability of 3DMB to more accurately detect and grade prostate cancer [1]. It remains to be seen, however, where cancers are being missed in standard office TRUS-guided procedures compared to $3 \mathrm{DMB}$ findings, and whether these missed cancers lead to diagnostic upgrades necessitating more aggressive treatment. An understanding of where prostate cancer is being missed by traditional 12-core TRUS-guided biopsy procedures carries implications for not only reducing understaging, but also reducing overtreatment if a more accurate and reliable diagnostic methodology is pursued. Thus, the aim of the research presented here is to evaluate the differences in cancer location obtained by 3DMB and TRUS biopsy methods in an effort to highlight potential trends in the location of missed cancers and cancer foci that result in upstaged and upgraded cancer.

\section{Methods}

Patients are seen at our institution for mapping biopsies due to recurrent negative biopsies in the presence of rising PSA, preference for watchful waiting but more risk assessment data, and/or pursuit of focal therapy (in which case $3 \mathrm{DMB}$ is standard of care). Our study is a Colorado Institutional Review Board (COMIRB) approved retrospective comparison of our 3DMB results with prior TRUS biopsy results for men aged 40-85 years with either low-risk organ confined prostate cancer or high suspicion for cancer who were seen at our institution between 2006 and 2009. Low-risk classification was based on regionally defined TRUS report demonstrating Gleason $3+4=7$ or lower, $\leq 50 \%$ tumor burden, and a PSA $<10 \mathrm{ng} / \mathrm{dL}$. 161 men met the inclusion criteria; of those, $68 \%(n=110)$ had mapping biopsies performed by urologist $\mathrm{AB}$ and the remaining $32 \%(n=$ 51) had mapping biopsies performed by urologist EDC. Approximately half of the TRUS biopsies were performed in house and half were performed at tertiary care centers. Most patients had TRUS and $3 \mathrm{DMB}$ performed within the same year; however, the maximum duration allowed between biopsies was 3 years.

As $3 \mathrm{DMB}$ coordinates are not readily translated into the zones typically used in TRUS reports, we divided $3 \mathrm{DMB}$ coordinates into six locations for comparison: Left Apex (LA), Left Mid (LM), Left Base (LB), Right Apex (RA), Right Mid (RM), and Right Base (RB). These are homologous with the locations reported in standard 12-core TRUS biopsies. Collected data included age, prostate size, number of days between biopsy procedures, use of 5-alpha-reductase inhibitors (5-ARIs), number of cores taken, and zones of the prostate positive for cancer.

Transperineal mapping biopsy was performed based on the $5 \mathrm{~mm}$ grid method using triplane transrectal ultrasound with accessory probe stabilization under general anesthesia with patients in the high dorsal lithotomy position. The ultrasound has the capability to have stepwise linear analogous or equivalent to the brachytherapy model. Two gold fiducial markers we placed at the time of 3DMB serve as an orientation landmark for future potential diagnostic or treatment considerations. All specimens from the biopsy of the prostate were marked according to their topographical location within the prostate and sent individually for pathological reporting. Total operating time is on average 60 minutes, whereas $3 \mathrm{DMB}$ biopsy time itself ranges from 15 to 25 minutes. Statistical analysis was performed in SAS 9.2.

\section{Results}

Of the 161 men enrolled in this study, the mean age was $61.6 \pm 7.73$, and the mean duration between TRUS and $3 \mathrm{DMB}$ for each patient was $192.18 \pm 287.96$ days, the mean prostate size was $49.34 \pm 20.65$ grams at time of TRUS biopsy and $39.25 \pm 15.25$ grams at the time of 3 DMB. Prostate size at time of 3DMB ranged from 17 to 95 grams. Fifty-three subjects were taking 5-ARIs during the time between TRUS-guided biopsy and $3 \mathrm{DMB}$; the mean prostatic volume reduction among these patients was $8.01 \pm 15.2$ grams.

Mean number of cores taken for $3 \mathrm{DMB}$ was $61.4 \pm 22.69$, ranging between 27 and 124. The mean number of positive cores per mapping biopsy was $5.25 \pm 4.81$. The median positive number of zones per patient using TRUS-guided biopsy was 1 (IQR $=0.63$ to 2.13 ) compared to a median number of positive zones for $3 \mathrm{DMB}$ of 2 (IQR $=0.59$ to 6.10 ). The amount of positive zones found from $3 \mathrm{DMB}$ was greater and statistically significant than the amount of positive zones found from TRUS-guided biopsy (3.33 versus $1.38, P<$ 0.0001).

Following TRUS-guided biopsy, $75 \%$ of patients $(n=121)$ were found to have Gleason 6 cancer and $7.5 \%(n=12)$ were found to have Gleason 7 cancer. Following 3DMB, 54\% of patients $(n=87)$ were found to have Gleason 6 cancer, $25 \%$ 
TABLE 1: Gleason grade distribution.

\begin{tabular}{lcc}
\hline Gleason & TRUS & 3 DMB \\
\hline 0 & 28 & 24 \\
6 & 121 & 87 \\
7 & 12 & 40 \\
8 & 0 & 7 \\
9 & 0 & 3 \\
\hline
\end{tabular}

$(n=40)$ were found to have Gleason 7 cancer, $4 \%(n=7)$ were found to have Gleason 8 cancer, and $2 \%(n=3)$ were found to have Gleason 9 cancer (Table 1).

Twenty-eight patients (17.4\%) were found to be cancerfree following TRUS-guided biopsy compared to 24 (14.9\%) following 3DMB. Of the 24 patients who were reported cancer-free by $3 \mathrm{DMB}, 11$ had a prior positive TRUS-guided biopsy. Of the 28 patients who reported cancer-free by initial TRUS-guided biopsy, 18 were found to be cancer-positive on subsequent $3 \mathrm{DMB}$. Of the cancers missed by $3 \mathrm{DMB}, 12.9 \%$ were Gleason $3+4=7$, and $87.1 \%$ were Gleason $3+3=6$. Of the cancers missed by TRUS, $60.3 \%$ were Gleason $3+3=6$, $24.9 \%$ were Gleason $3+4=7$, $4.4 \%$ were Gleason $4+4=8$, and $2 \%$ was Gleason $4+5=9$.

There were 68 instances in which the LM zone was recorded cancer-positive by $3 \mathrm{DMB}$ in the face of prior negative TRUS-guided biopsy results, followed by 62 instances in the RM zone and 41 instances in the LA zone. Thus, most newly discovered cancers originated from the middle of the left lobe, middle of the right lobe, and apex of left lobe. Approximately one-half of all newly discovered cancerous zones were diagnosed as a result of anterior $3 \mathrm{DMB}$ core sampling. In our sample, 56 patients experienced an overall Gleason upgrade (34.8\%), with newly discovered Gleason 7 findings following a similar distribution pattern as newly discovered Gleason 6 findings. For cases where both biopsy methods found positive cores in a given zone, Gleason upgrades occurred most frequently in the middle left lobe. The TRUS cancer-positive zones that were most often unconfirmed for cancer by $3 \mathrm{DMB}$ were the base of the right lobe and the base of the left lobe. In cases where unilateral cancer was found on TRUS biopsy, 3DMB reported cancer on the contralateral side of the prostate for approximately $28.5 \%$ $(n=46)$ of patients (Tables 2 and 3$)$.

Of the 161 men enrolled, one patient experienced an episode of atrial fibrillation during anesthesia. One patient experienced contact dermatitis probably due to the antiseptic used during the mapping biopsy. One patient with a history of DVTs presented to the clinic 8 days after his mapping biopsy with a DVT. One patient complained of incontinence the day after his mapping procedure, and this was resolved with oxybutynin. Complication rates for these procedures have been described previously [13].

\section{Discussion}

It has been demonstrated previously that $3 \mathrm{DMB}$ provides a more accurate depiction of prostate tumor burden and the presence of contralateral tumors due to more extensive sampling, methodical biopsy spacing, and access to the anterior region of the prostate $[13,14]$. Our data demonstrates that the majority of prostate cancers missed by TRUS but detected by $3 \mathrm{DMB}$ as well as the majority of cancer stage upgrades appear to originate in the middle of the left and right zones of the prostate, with a significant proportion of newly discovered cancers derived from anterior core sampling.

The data suggest poor agreement between 12-core TRUS biopsy and $3 \mathrm{DMB}$ results with regard to staging and grading of prostate cancer, most notably in the aforementioned regions of the prostate. While our data demonstrate a high relative positive predictive and negative predictive value for cancer presence versus no presence when comparing TRUS biopsy to $3 \mathrm{DMB}$, the lack of concordance regarding location of cancer, findings of contralateral cancer, and discovery of greater amounts and higher grades of cancer raise concern. The relatively high rates of cancer upgrading upon $3 \mathrm{DMB}$ are in accordance with previous studies [13]. These results also support conclusions reached from whole mount radical prostatectomy specimen studies and repeat biopsy studies which demonstrated higher levels of anterior region cancers amongst patients with previous negative TRUS biopsies [15]. Research performed by Duffield et al. on RP specimens from patients for whom AS failed also suggests that increased sampling in the anterior region is necessary [16].

The importance of anterior core sampling follows, given the difficulties associated with accessing anterior regions of the prostate using the transrectal approach, along with the fact that approximately $1 / 5$ of carcinomas are found to lie anterior to the urethra in RP specimens [17]. MRI imaging modalities have progressed through the years in relation to prostate cancer detection and evaluation and this may also assist in anterior zone sampling $[18,19]$. Current studies seem to demonstrate its potential; however, the data is still preliminary [20].

While other studies evaluating cancer location have employed the use of sectioned specimens, we did not feel that this was appropriate in our circumstances due to the volume loss and change in core position upon prostatectomy. Limitations of this study surround the consistency of TRUS biopsy, given the fact that approximately half of the TRUS biopsy records included in this study were in house, whereas the other half came from tertiary centers. Another concern regards the use of 5-alpha-reductase-inhibitors (5-ARIs), which were employed to shrink the volume of the prostate when the pubic arch obstructed transperinneal access. Some research suggests that 5-ARIs, by shrinking the prostate, actually highlight the presence of high grade disease, thus heightening the sensitivity of diagnostic tests like 3DMB [21]. The specific effect of 5-ARIs on the diagnostic location and grade of prostate cancer as determined by $3 \mathrm{DMB}$ has yet to be determined. Systemic improvements in prostate cancer detection and AS enrollment must come with sensible systemic changes, and a large component of this will be cost. Thus, a cost-effectiveness analysis of prostate cancer diagnostic techniques will serve greatly in the effort to avoid overdiagnosis and overtreatment. It should be noted that repeat of TRUS biopsy may yield additional cancer at the 
TABLE 2: 3DMB and TRUS-guided biopsy characteristics.

\begin{tabular}{lcc}
\hline & TRUS & 3DMB \\
\hline Total cores taken per patient & 12 & \\
Number of positive cores per patient & $1.38( \pm 1.21)$ & Patients \\
& Patients & $71 / 161(44.1 \%)$ \\
LA positive & $55 / 161(34.2 \%)$ & $109 / 161(67.7 \%)$ \\
LM positive & $48 / 161(29.8 \%)$ & $37 / 161(23.0 \%)$ \\
LB positive & $40 / 161(24.8 \%)$ & $54 / 161(33.5 \%)$ \\
RA positive & $43 / 161(26.7 \%)$ & $104 / 161(64.4 \%)$ \\
RM positive & $52 / 161(32.3 \%)$ & $32 / 161(19.9 \%)$ \\
RB positive & $39 / 161(24.2 \%)$ & \\
\hline
\end{tabular}

TABLE 3: Newly discovered cancers and cancer upgrades.

\begin{tabular}{|c|c|c|c|c|c|c|}
\hline & LA & LM & LB & RA & $\mathrm{RM}$ & $\mathrm{RB}$ \\
\hline $\begin{array}{l}\text { Instances of cancer positive cores coming } \\
\text { from TRUS-determined cancer negative } \\
\text { zones (total instances }=244 \text { ) }\end{array}$ & $41(16.8 \%)$ & $68(27.9 \%)$ & $19(7.8 \%)$ & $35(14.3 \%)$ & $62(25.4 \%)$ & $19(7.8 \%)$ \\
\hline $\begin{array}{l}\text { Proportion of newly cancerous zones } \\
\text { resulting from anterior } 3 \mathrm{DMB} \text { cores } \\
\text { (total instances }=108 \text { ) }\end{array}$ & $17(41.5 \%)$ & $28(41.2 \%)$ & $8(42.1 \%)$ & $17(48.6 \%)$ & $25(40.3 \%)$ & $13(68.4 \%)$ \\
\hline $\begin{array}{l}\text { Instances of positive Gleason } 7 \text { cores } \\
\text { (total instances }=178 \text { ) }\end{array}$ & $32(18 \%)$ & $43(24.7 \%)$ & $13(7.3 \%)$ & $24(13.5 \%)$ & $56(31.5 \%)$ & $10(5.6 \%)$ \\
\hline $\begin{array}{l}\text { Gleason upgrade instances by zone } \\
(3+4=7,4+3=7, \text { or higher }) \text { by zone }(\mathrm{s}) \\
(N=56, \text { total instances }=98)\end{array}$ & $18(18.4 \%)$ & $28(28.6 \%)$ & $4(4.1 \%)$ & $14(14.3 \%)$ & $28(28.6 \%)$ & $6(6 \%)$ \\
\hline $\begin{array}{l}\text { Instances of positive Gleason } 7 \text { cores for } \\
\text { zones which both biopsy methods reported } \\
\text { as cancer positive }\end{array}$ & 3 & 8 & 1 & 2 & 4 & 0 \\
\hline $\begin{array}{l}\text { Instances of unconfirmed cancer by zone } \\
\text { upon } 3 \mathrm{DMB} \text { (total instances }=99 \text { ) }\end{array}$ & $17(17.2 \%)$ & $8(8 \%)$ & $20(20.2 \%)$ & $18(18.2 \%)$ & $12(12.1 \%)$ & $24(24.3 \%)$ \\
\hline
\end{tabular}

same rate of $3 \mathrm{DMB}$, however localization is more difficult to determine and advancements in anterior sampling may still be necessary.

As of this study, more than 700 mapping biopsies have been performed at our institution without any documented urinary retention referring to the time period following the first 48 hours after the procedure. All patients have a catheter for 24-48 hours following the procedure, the duration of which is determined by the operator who takes into account preprocedure lower urinary tract symptoms. Pre-3DMB urinary tract symptoms related to $\mathrm{BPH}$ returned to baseline within a week. Patients who undergo $3 \mathrm{DMB}$ are advised to take tamsulosin hydrochloride 10 days after the procedure or to continue existing $\mathrm{BPH}$ medications. In our experience, the number of mapping biopsy cores did not affect postoperative recovery; however, patients with preexisting lower urinary tract symptoms had longer recovery times.

\section{Conclusion}

Overtreatment is an increasingly important issue in the field of prostate biopsy and more accurate cancer detection has the potential to increase the utility of AS and reduce the amount of overtreatment, while at the same time providing appropriate treatment for those who may go understaged due to insufficient sampling. The majority of prostate cancers missed by TRUS but detected by $3 \mathrm{DMB}$ as well as most cancer upgrades appear to originate in the middle of the left lateral and right lateral zones of the prostate, specifically in anterior regions. Attention should be given to this region of the prostate when evaluating for prostate cancer in order to improve biopsy accuracy and avoid undersampling. The potential for missed cancer in this region is significant, which suggests that efforts made to address anterior prostate sampling may lead to improved outcomes and enrollment in AS.

\section{References}

[1] A. B. Barqawi, K. Krughoff, and K. Eid, "Current challenges in prostate cancer management and the rationale behind targeted focal therapy," Advances in Urology, vol. 2012, Article ID 862639, 7 pages, 2012.

[2] P. C. Albertsen, "Is screening for prostate cancer with prostate specific antigen an appropriate public health measure?" Acta Oncologica, vol. 44, no. 3, pp. 255-264, 2005.

[3] P. C. Albertsen, J. A. Hanley, and J. Fine, "20-Year outcomes following conservative management of clinically localized prostate cancer," Journal of the American Medical Association, vol. 293, no. 17, pp. 2095-2101, 2005. 
[4] L. Klotz, L. Zhang, A. Lam, R. Nam, A. Mamedov, and A. Loblaw, "Clinical results of long-term follow-up of a large, active surveillance cohort with localized prostate cancer," Journal of Clinical Oncology, vol. 28, no. 1, pp. 126-131, 2010.

[5] N. Lawrentschuk and L. Klotz, "Active surveillance for low-risk prostate cancer: an update," Nature Reviews Urology, vol. 8, no. 6, pp. 312-320, 2011.

[6] M. R. Cooperberg, J. M. Broering, P. W. Kantoff, and P. R. Carroll, "Contemporary trends in low risk prostate cancer: risk assessment and treatment," Journal of Urology, vol. 178, no. 3, pp. S14-S19, 2007.

[7] M. R. Cooperberg, J. M. Broering, and P. R. Carroll, "Time trends and local variation in primary treatment of localized prostate cancer," Journal of Clinical Oncology, vol. 28, no. 7, pp. $1117-1123,2010$.

[8] R. Chou, J. M. Croswell, T. Dana et al., "Screening for prostate cancer: a review of the evidence for the U.S. preventive services task force," Annals of Internal Medicine, vol. 155, no. 11, pp. 762771, 2011.

[9] V. Iremashvili, M. Manoharan, D. L. Rosenberg, and M. S. Soloway, "Biopsy features associated with prostate cancer progression in active surveillance patients: comparison of three statistical models," British Journal of Urology, vol. 111, no. 4, pp. 574-579, 2013.

[10] M. C. Large and S. E. Eggener, "Active surveillance for low-risk localized prostate cancer," Oncology, vol. 23, no. 11, pp. 974-979, 2009.

[11] A. S. Duffield, T. K. Lee, H. Miyamoto, H. B. Carter, and J. I. Epstein, "Radical prostatectomy findings in patients in whom active surveillance of prostate cancer fails," Journal of Urology, vol. 182, no. 5, pp. 2274-2279, 2009.

[12] K. Lees, M. Durve, and C. Parker, "Active surveillance in prostate cancer: patient selection and triggers for intervention," Current Opinion in Urology, vol. 22, no. 3, pp. 210-215, 2012.

[13] A. B. Barqawi, K. O. Rove, S. Gholizadeh, C. I. O’Donnell, H. Koul, and E. D. Crawford, "The role of 3-dimensional mapping biopsy in decision making for treatment of apparent early stage prostate cancer," Journal of Urology, vol. 186, no. 1, pp. 80-85, 2011.

[14] J. L. Dominguez-Escrig, S. R. C. McCracken, and D. Greene, "Beyond diagnosis: evolving prostate biopsy in the era of focal therapy," Prostate Cancer, vol. 2011, Article ID 386207, 11 pages, 2011.

[15] A. R. Patel and J. S. Jones, "Optimal biopsy strategies for the diagnosis and staging of prostate cancer," Current Opinion in Urology, vol. 19, no. 3, pp. 232-237, 2009.

[16] A. S. Duffield, T. K. Lee, H. Miyamoto, H. B. Carter, and J. I. Epstein, "Radical prostatectomy findings in patients in whom active surveillance of prostate cancer fails," Journal of Urology, vol. 182, no. 5, pp. 2274-2279, 2009.

[17] S. R. J. Bott, M. P. A. Young, M. J. Kellett, and M. C. Parkinson, "Anterior prostate cancer: is it more difficult to diagnose?" British Journal of Urology, vol. 89, no. 9, pp. 886-889, 2002.

[18] M. A. Haider, T. H. Van Der Kwast, J. Tanguay et al., "Combined T2-weighted and diffusion-weighted MRI for localization of prostate cancer," American Journal of Roentgenology, vol. 189, no. 2, pp. 323-328, 2007.

[19] P. Puech, N. Betrouni, N. Makni, A.-S. Dewalle, A. Villers, and L. Lemaitre, "Computer-assisted diagnosis of prostate cancer using DCE-MRI data: design, implementation and preliminary results," International Journal of Computer Assisted Radiology and Surgery, vol. 4, no. 1, pp. 1-10, 2009.
[20] E. K. Vos, G. J. S. Litjens, T. Kobus et al., "Assessment of prostate cancer aggressiveness using dynamic contrast-enhanced magnetic resonance imaging at 3T,' European Urology, vol. 64, no. 3, pp. 448-455, 2013.

[21] R. J. Hamilton and S. J. Freedland, "5- $\alpha$ reductase inhibitors and prostate cancer prevention: where do we turn now?" BMC Medicine, vol. 9, article 105, 2011. 


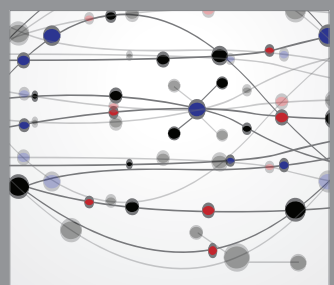

The Scientific World Journal
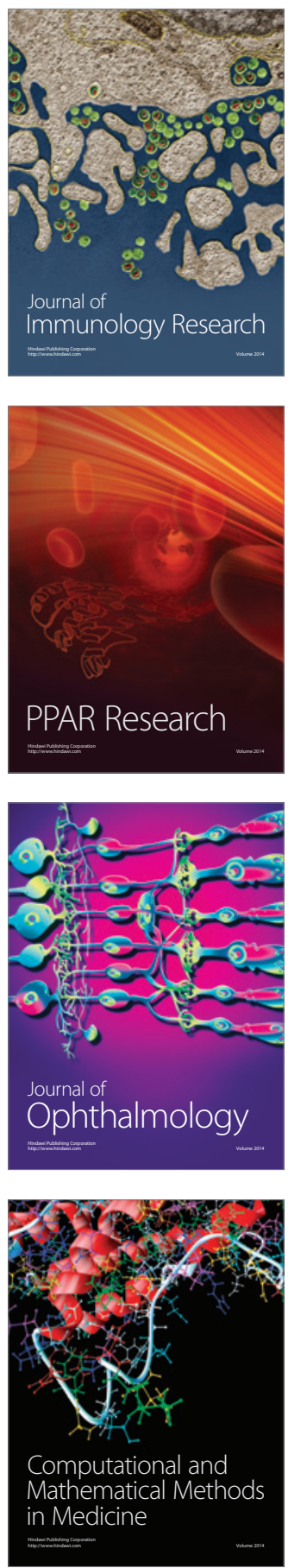

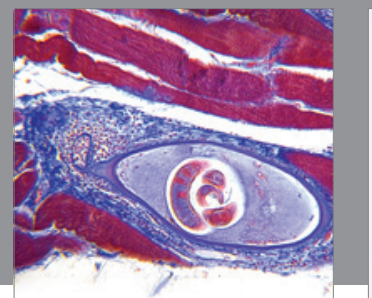

Gastroenterology

Research and Practice
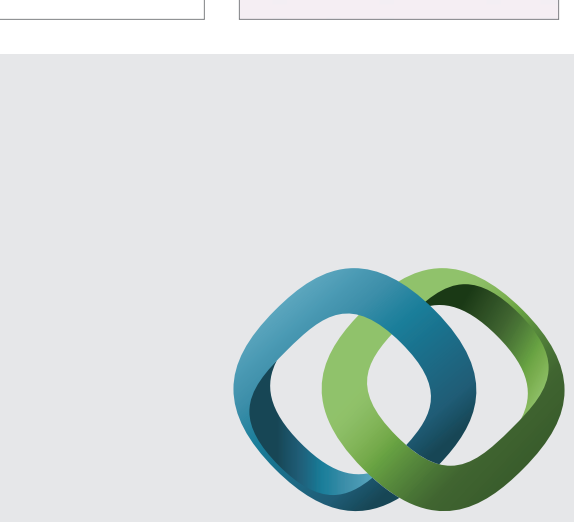

\section{Hindawi}

Submit your manuscripts at

http://www.hindawi.com
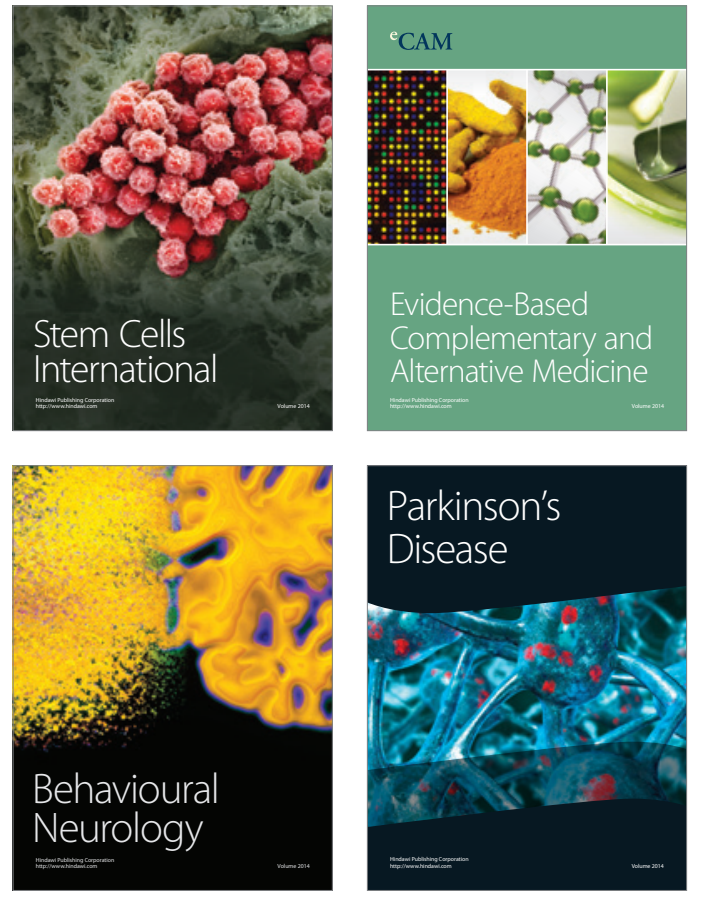
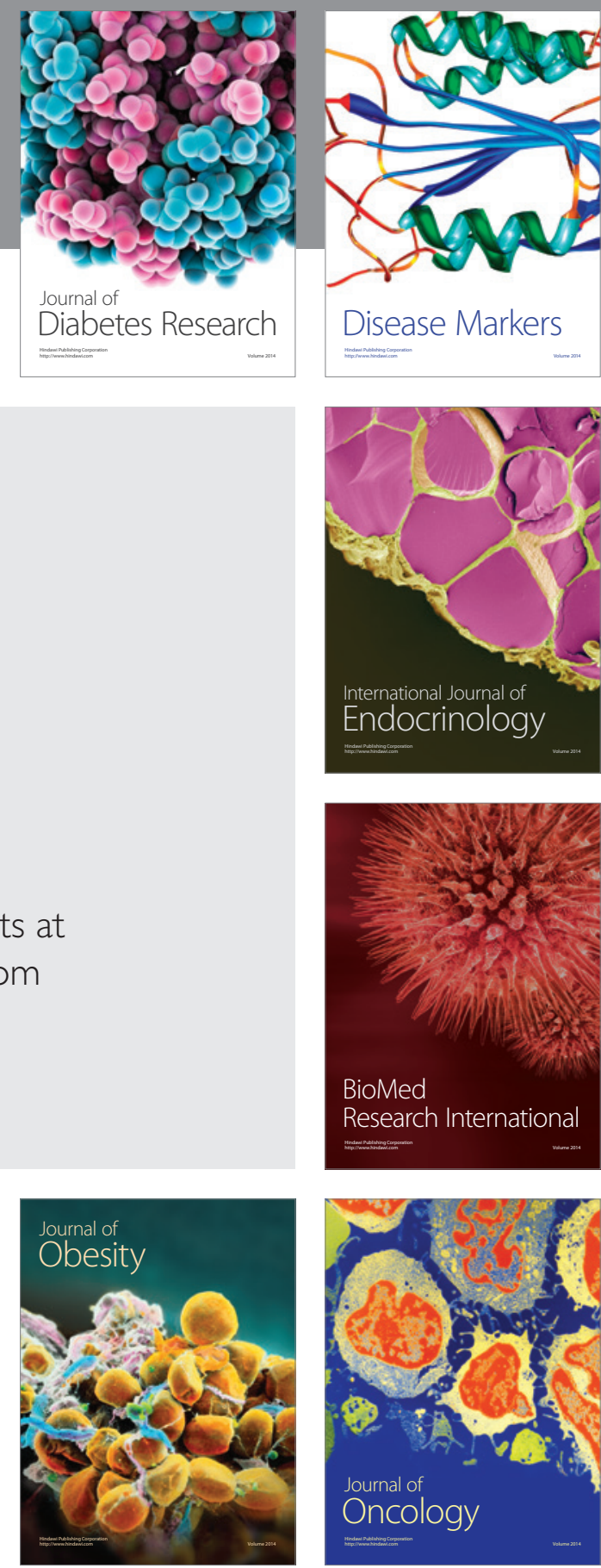

Disease Markers
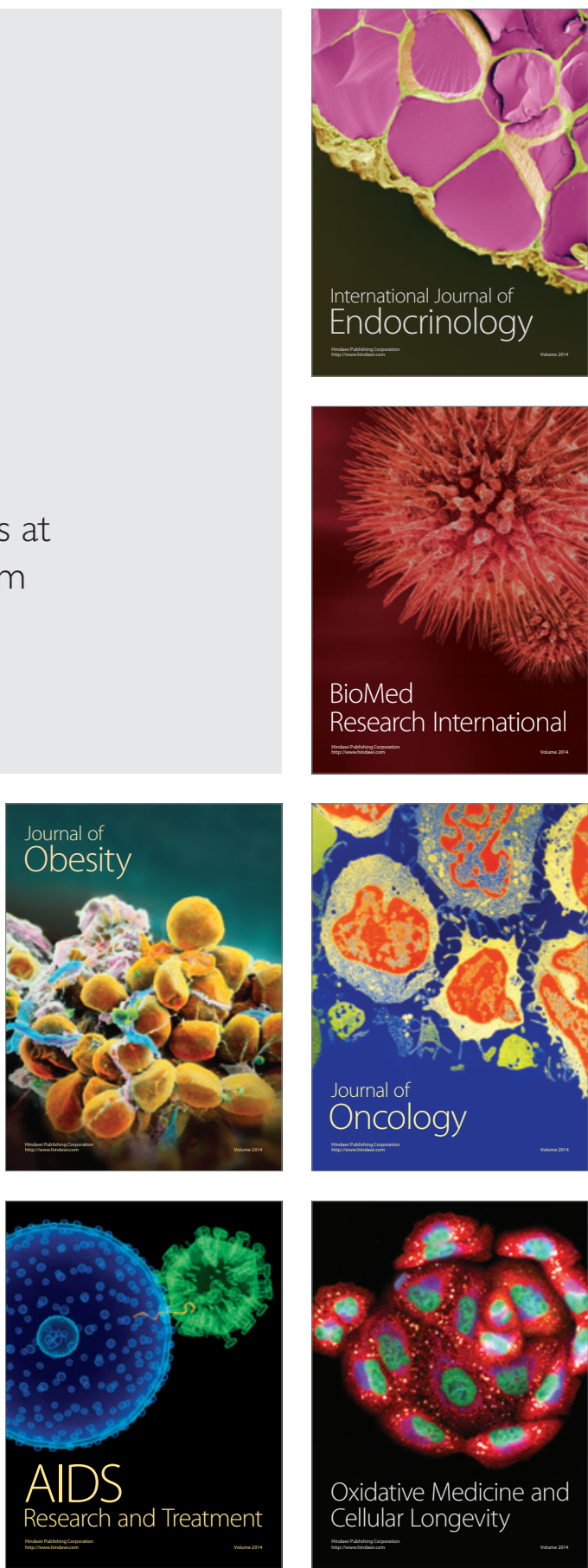